\title{
The Heavy Photon Search Experiment
}

\author{
Maurik Holtrop* \\ University of New Hampshire, Durham, NH, USA \\ E-mail: maurik.holtrop@unh.edu
}

In recent years much interest has been given to new physics models which have hidden sectors with massive extra U(1) gauge bosons, so-called heavy photons (or dark photons). Such theories could account for Dark Matter annihilation and explain excess electrons and positrons in cosmic rays. The Heavy Photon Search Experiment (HPS) at Jefferson Lab is a new experiment designed to look for these heavy photons in the mass range $20-1000 \mathrm{MeV}$ that couple to electrons through kinetic mixing with couplings $\alpha^{\prime} / \alpha$ in the range $10^{-5}$ to $10^{-10}$. The HPS will search for the $e^{+} e^{-}$or $\mu^{+} \mu^{-}$decay of the heavy photon, possibly with a displaced vertex, using a compact forward spectrometer, which employs silicon microstrip detectors for vertexing and tracking, and a PbWO4 electromagnetic calorimeter for fast triggering and electron identification, and a muon detector for muon identification. The design, performance, and results from the test run apparatus will be discussed, along with the collaboration's plans for future construction and data taking.

International Conference on the Structure and the Interactions of the Photon including the 20th International Workshop on Photon-Photon Collisions and the International Workshop on High Energy Photon Linear Colliders

20 - 24 May 2013

Paris, France

${ }^{*}$ Speaker. 


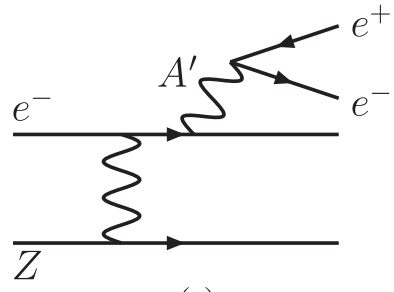

(a)

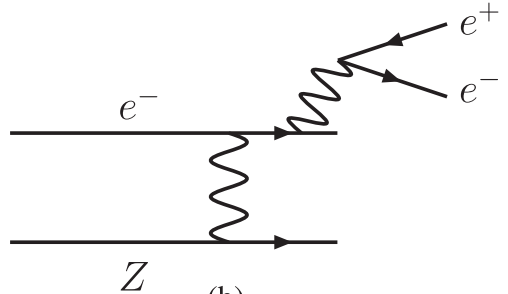

(b)

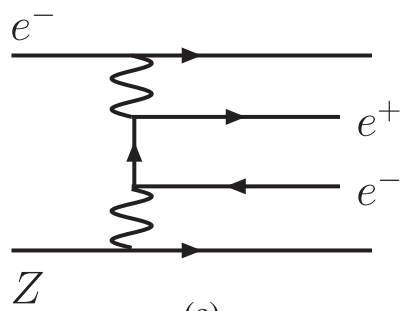

(c)

Figure 1: Left (a): Radiative $A^{\prime}$ production by an incoming electron off a target nucleus with charge $Z$. The $A^{\prime}$ subsequently decays to an electron-positron pair. For larger mass $A^{\prime}$, with $m_{A^{\prime}}>2 m_{\mu}$, the decay will also go to a $\mu^{+} \mu^{-}$pair, and for $m_{A^{\prime}}>2 m_{\pi}$ to a $\pi^{+} \pi^{-}$pair. Center and Right: Background due to QED trident production, (b) radiative process and (c) Bethe-Heitler process.

\section{Introduction}

It is by now well established that the universe consists of approximately $26 \%$ Dark Matter (DM), and that there are no candidates for this DM in the Standard Model (SM) of particle interactions [1]. A particle based explanation for DM would thus require extensions of the SM, exhibiting hidden sectors of particles that interact only very weakly with SM particles. Such Beyond the Standard Model (BSM) theories often have additional $U(1)$ gauge symmetries with associated gauge bosons, also known as heavy photons, dark photons, or $A^{\prime}$ [2, 3, 4]. As was pointed out some time ago by Holdom [5], this new vector particle will kinematically mix with the SM photon through the interaction with massive fields. This mixing can be recast as a coupling of the $A^{\prime}$ to electric charge $\varepsilon e$, where the expected strength of $\alpha^{\prime} / \alpha=\varepsilon^{2}$ is in the range $10^{-5}$ to $10^{-10}$.

Several recent theoretical models have explored the interactions of an $A^{\prime}$ with dark matter in a hidden sector $[6,7,8,9]$. These theories favor a mass for the $A^{\prime}$ derived from the weak scale, in the $\mathrm{MeV}$ to $\mathrm{GeV}$ range, which could be used to explain several observed astrophysical phenomena [10, 11], including the excess of positrons observed by Pamela [12], Fermi-LAT [13] and AMS [14]. Furthermore an $A^{\prime}$ in the lower part of this mass region could account for the anomalous magnetic moment of the muon [15].

The parameters space of $\alpha^{\prime} / \alpha$ versus $m_{A^{\prime}}$ is still only partially constrained by cosmological phenomena and experiment [4], leaving the interesting region in the $\mathrm{MeV}$ to $\mathrm{GeV}$ mass range largely unexplored. This region can be ideally accessed using high-intensity electron beams, using a high $Z$ fixed-target and either dedicated or existing spectrometers [16]. In such an experiment the heavy photons are produced in a process similar to bremsstrahlung, where the electron looses most of its energy to a radiated $A^{\prime}$ particle, which will predominantly be produced along the direction of the beam, as is illustrated in Figure 1. This $A^{\prime}$ will then decay to an $e^{+} e^{-}$pair (or if sufficiently, heavy a $\mu^{+} \mu^{-}$or $\pi^{+} \pi^{-}$pair), which will be highly boosted in the direction of the beam, necessitating detection at very small scattering angles. An experiment will thus require very high luminosity to overcome the small production cross-section, and a very forward, high rate, high resolution spectrometer to detect the produced lepton pair. The $A^{\prime}$ signal will then be searched for as a small, narrow, peak in the $e^{+} e^{-}$invariant mass on a smooth background. The main background to such an experiment will be QED trident production, the Bethe-Heitler and radiative production 
of electron-positron pairs. The radiative term of this background is indistinguishable from the main $A^{\prime}$ production process, but the dominant Bethe-Heitler background can be suppressed using kinematic selection, either in the experimental trigger, or through the spectrometer settings. This works because the radiative tridents, and hence the $A^{\prime}$ signal, are produced very forward with the electronpositron pair carrying most of the beam energy, while the recoiling electron is soft and scatters at a wide angle. In contrast, the Bethe-Heitler process favors asymmetric configurations with one energetic, forward electron or positron and the other constituent of the pair much softer. See the appendix in [16] for a more detailed explanation. Another way to suppress both backgrounds compared to the $A^{\prime}$ signal is to look for a displaced vertex for the $e^{+} e^{-}$pair. Since the decay length of the $A^{\prime}$ is approximately $\gamma c \tau \approx 1 \mathrm{~mm}\left(\frac{\gamma}{10}\right)\left(10^{-8} \frac{\alpha}{\alpha^{\prime}}\right)\left(\frac{100 \mathrm{MeV}}{m_{A^{\prime}}}\right)$, for small coupling constants and moderate to small masses the decay length becomes appreciable. This allows for a cut in the data analysis on the vertex of the $e^{+} e^{-}$pair, reducing the prompt background significantly.

\section{The Heavy Photon Search Experiment}

The Heavy Photon Search experiment (HPS) $[17,18,19]$ will search for $A^{\prime}$ particles in a mass range of $20 \mathrm{MeV} / \mathrm{c}^{2}<m_{A^{\prime}}<1000 \mathrm{MeV} / \mathrm{c}^{2}$, with coupling strengths $\varepsilon^{2}=\alpha^{\prime} / \alpha \gtrsim 10^{-5}$ to $10^{-10}$. This experiment will use a new, dedicated, compact forward spectrometer in Hall B at Jefferson Laboratory. The experiment will use the continuous electron beam with energies ranging from $1.1 \mathrm{GeV}$ to $6.6 \mathrm{GeV}$ and currents up to $500 \mathrm{nA}$ on a thin tungsten target of radiation lengths up to $0.25 \% X_{0}$. An intriguing additional physics signal for the HPS experiment will be the possible discovery of true muonium, an $\mu^{+} \mu^{-}$bound state that has so far eluded detection [20, 21]

The HPS experimental setup, shown in Fig. 2, uses a three magnet chicane, with the middle dipole magnet, the Hall-B pair spectrometer dipole, used as the analyzing magnet for the spectrometer. Inside this magnet a vacuum chamber is fitted with the target on a moving target ladder at the upstream edge. This vacuum chamber also houses the Silicon Vertex Tracker (SVT) [22], which consists of six measurement layers, each consisting of two pairs of silicon strip detectors. All the HPS detector elements are split into a top and bottom half with a gap in between, the "dead zone", extending a scattering angle of $15 \mathrm{mrad}$. This gap, which is only $\pm 1.5 \mathrm{~mm}$ at the location of the first sensor, allows the primary and degraded electron beam to pass through without further interaction. The first three tracking layers are optimized for obtaining an excellent vertex determination $(\Delta z \approx 1 \mathrm{~mm})$, and are placed at 10,20 and $30 \mathrm{~cm}$ from the target, with the second layer at a $100 \mathrm{mrad}$ stereo angle. The next three layers of the tracker are optimized for missing mass resolution $(\delta m / m \approx 1 \%)$, and are placed at 50,70 and $90 \mathrm{~cm}$ from the target at a $50 \mathrm{mrad}$ stereo angle. The 23,004 channels of the SVT will be continuously read out at $40 \mathrm{MHz}$ using the APV25 readout chip, which will read six data samples per hit, allowing for a timing resolution of $\approx 2 \mathrm{~ns}$ and a $\mathrm{S} / \mathrm{N}>25$, resulting in a spacial resolution of $\approx 6 \mu \mathrm{m}$.

The tracking volume is followed by an Electromagnetic Calorimeter (Ecal), which is used for particle identification and for triggering the experiment. The Ecal consisting of $442 \mathrm{PbWO}_{4}$ crystals arranged in 5 layers each for the top and bottom half of the detector. In between the two halves is a vacuum box for the electron beam, which has been carefully designed to minimize interactions of the multiple scattered and degraded electron beam. Each Ecal crystal is read out by an avalanche photodiode, which is connected to a pulse shaping preamplifier whose signal is digitized by a JLab 


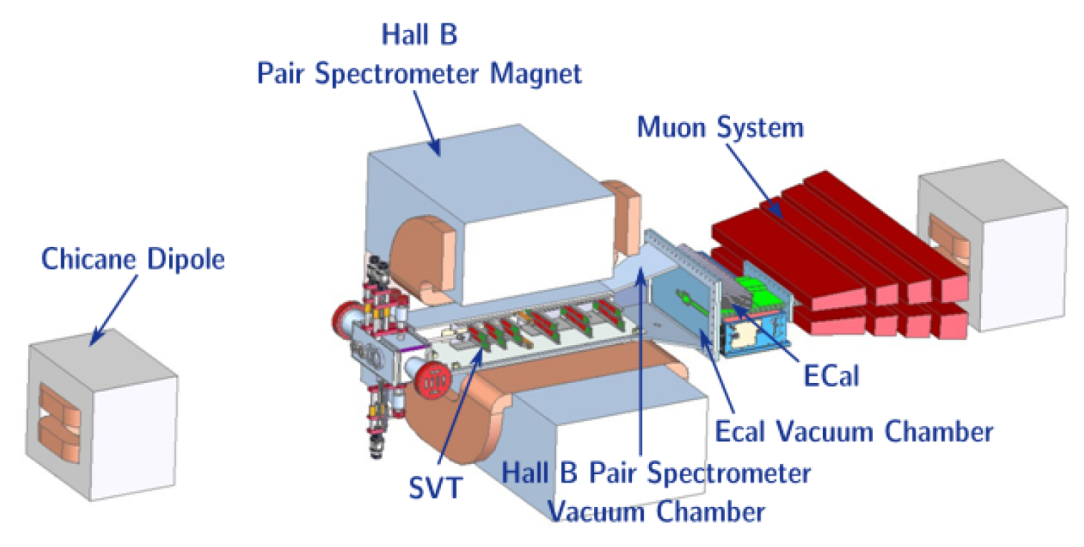

Figure 2: Engineering layout of the full HPS detector, showing the three dipole magnets of the chicane, the Silicon Vertex Tracker (SVT), the Electromagnetic Calorimeter (Ecal) and the Muon System.

FADC250, a $250 \mathrm{MHz}$ flash ADC. From there the pulse height and timing signals of the individual crystals are combined into clusters in a Field Programmable Gate Array (FPGA), and then passed on to the trigger logic. The trigger logic is also programmed into an FPGA and is capable of using the energy and location of the clusters to select between the signal and radiative events and those caused by accidental coincidences. This trigger logic reduces the trigger rate from close to $2 \mathrm{MHz}$ down to a more manageable $35 \mathrm{kHz}$.

Behind the Ecal there will be a muon detector, build from alternating layers of iron absorber and plastic scintillator. The design details of this detector are still being optimized.

A simplified version of the full HPS detector was constructed and took data during a test run in May 2012. This was just before the accelerator shutdown and subsequent upgrade from $6 \mathrm{GeV}$ to $12 \mathrm{GeV}$, and due to scheduling constraints there was no dedicated beam time available with an electron beam. Instead, data was taken parasitically using a photon beam incident on a thin gold converter foil serving as a target upstream of the detector. This test run successfully demonstrated the technical feasibility of the HPS detector concept and confirmed that the Monte Carlo estimates of backgrounds and trigger rates were accurate.

\section{Experimental Reach}

The experimental reach of the HPS experiment at $2 \sigma$ significance is shown in the contours of Fig. 3, along with some existing constraints from previous experiments. The shaded areas show excluded regions from several beam dump experiments with sensitivity for heavy photons, including E141 [23], E774 [24], Orsay [25], and U70 [26], and the reanalysis of collider experiments from BaBar [27] and KLOE [28]. The measurement of the anomalous magnetic moment of the electron [29] also sets a limit, indicated withe the $a_{e}$ label. The anomalous magnetic moment of the muon gives both an excluded region $\left(a_{\mu \pm 5 \sigma}\right)$ and one that would be favored by a heavy photon explanation of the deviation of $g_{\mu}-2$ from the expected Standard Model value [15, 30] (green band). The recent test run from APEX [31] and MAMI [32] are also shown as shaded regions. The dashed line labelled "HPS Test" shows the reach of a two week commissioning run expected in 2015 , with one week of beam at $1.1 \mathrm{GeV}$ and one week at $2.2 \mathrm{GeV}$, while the solid red line labelled 


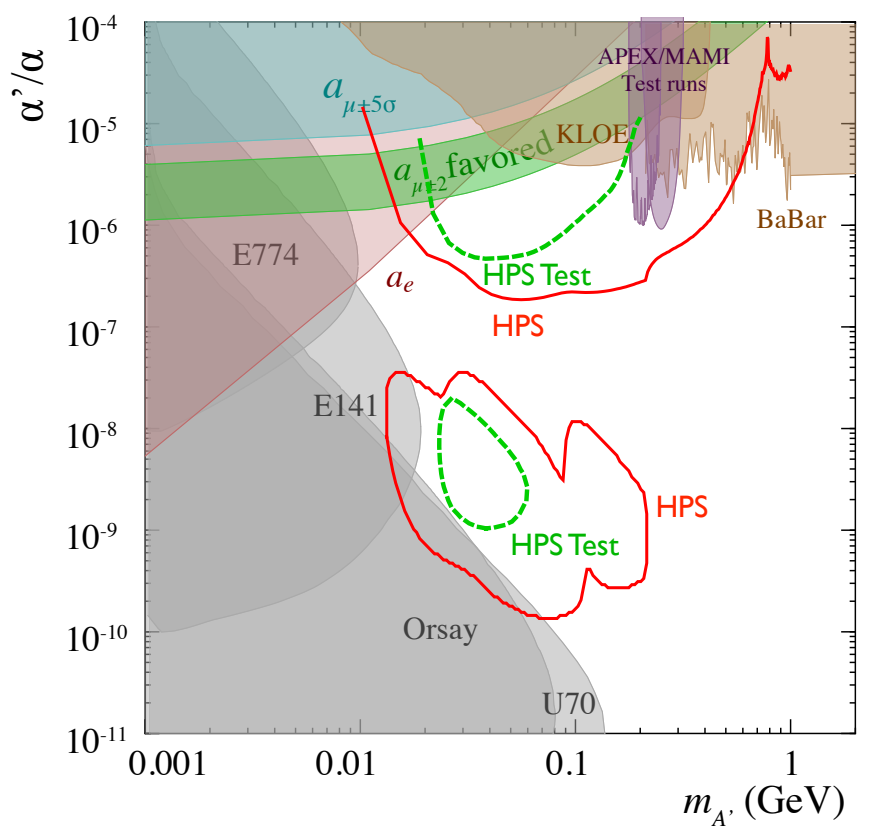

Figure 3: The estimated reach of the HPS experiment at $2 \sigma$ significance along with existing constraints (see text). The HPS reach calculations assume running at $1.1 \mathrm{GeV}$ and $2.2 \mathrm{GeV}$ for one week each in 2014/2015, labelled "HPS Test", and running at $2.2 \mathrm{GeV}$ and $6.6 \mathrm{GeV}$ for three weeks each in 2016, labelled "HPS".

"HPS" is the reach for three weeks of running each at $2.2 \mathrm{GeV}$ and $6.6 \mathrm{GeV}$, expected to start in 2017. The top regions are obtained from a "bump hunt" in the data, while the "island" below, at lower coupling strengths, is obtained by using a vertex cut as well. The HPS experiment has a total of 180 days of running approved, so a significant amount of data will still be taken after 2017 .

\section{Conclusions}

The HPS experiment has shown its technical feasibility in a recent test run. The HPS collaboration is now upgrading their test detector, which will be installed in time for a commissioning run in 2015, with additional running in 2017. The experiment has excellent sensitivity to search for of a heavy photon.

\section{Acknowledgments}

The work presented here is that of the Heavy Photon Search (HPS) Collaboration. The author gratefully acknowledges the support from DOE under contract no. DE-FG02-88ER40410.

\section{References}

[1] P. A. R. Ade et al. [Planck Collaboration], arXiv:1303.5062 [astro-ph.CO].

[2] P. Candelas, G. T. Horowitz, A. Strominger and E. Witten, Nucl. Phys. B 258, 46 (1985). 
[3] S. Andreas, M. D. Goodsell and A. Ringwald, Phys. Rev. D 87, 025007 (2013) [arXiv:1109.2869 [hep-ph]].

[4] J. Jaeckel and A. Ringwald, Ann. Rev. Nucl. Part. Sci. 60, 405 (2010) [arXiv:1002.0329 [hep-ph]].

[5] B. Holdom, Phys. Lett. B 166, 196 (1986).

[6] N. Arkani-Hamed, D. P. Finkbeiner, T. R. Slatyer and N. Weiner, Phys. Rev. D 79, 015014 (2009) [arXiv:0810.0713 [hep-ph]].

[7] M. Pospelov and A. Ritz, Phys. Lett. B 671, 391 (2009) [arXiv:0810.1502 [hep-ph]].

[8] N. Arkani-Hamed and N. Weiner, JHEP 0812, 104 (2008) [arXiv:0810.0714 [hep-ph]].

[9] C. Cheung, J. T. Ruderman, L. -T. Wang and I. Yavin, Phys. Rev. D 80, 035008 (2009) [arXiv:0902.3246 [hep-ph]].

[10] R. Essig, J. Kaplan, P. Schuster and N. Toro, [arXiv:1004.0691 [hep-ph]].

[11] I. Cholis, D. P. Finkbeiner, L. Goodenough and N. Weiner, JCAP 0912, 007 (2009) [arXiv:0810.5344 [astro-ph]].

[12] O. Adriani et al. [PAMELA Collaboration], Phys. Rev. Lett. 106, 201101 (2011) [arXiv:1103.2880 [astro-ph.HE]].

[13] M. Ackermann et al. [Fermi LAT Collaboration], Phys. Rev. Lett. 108, 011103 (2012) [arXiv:1109.0521 [astro-ph.HE]].

[14] M. Aguilar et al. [AMS Collaboration], Phys. Rev. Lett. 110, no. 14, 141102 (2013).

[15] M. Pospelov, Phys. Rev. D 80, 095002 (2009) [arXiv:0811.1030 [hep-ph]].

[16] J. D. Bjorken, R. Essig, P. Schuster and N. Toro, Phys. Rev. D 80, 075018 (2009) [arXiv:0906.0580 [hep-ph]].

[17] The HPS Collaboation, “The Heavy Photon Search Experiment at Jefferson Laboratory”, Proposals, https://confluence.slac.stanford.edu/display/hpsg/HPS+Proposals

[18] P. H. Adrian, arXiv:1301.1103 [physics.ins-det].

[19] O. Moreno, arXiv:1310.2060 [physics.ins-det].

[20] S. J. Brodsky and R. F. Lebed, Phys. Rev. Lett. 102, 213401 (2009) [arXiv:0904.2225 [hep-ph]].

[21] A. Banburski and P. Schuster, Phys. Rev. D 86, 093007 (2012) [arXiv:1206.3961 [hep-ph]].

[22] T. K. Nelson [HPS Collaboration], PoS Vertex 2012, 032 (2013).

[23] E. M. Riordan, M. W. Krasny, K. Lang, P. De Barbaro, A. Bodek, S. Dasu, N. Varelas and X. Wang et al., Phys. Rev. Lett. 59, 755 (1987).

[24] A. Bross, M. Crisler, S. H. Pordes, J. Volk, S. Errede and J. Wrbanek, Phys. Rev. Lett. 67, 2942 (1991).

[25] S. Andreas, C. Niebuhr and A. Ringwald, Phys. Rev. D 86, 095019 (2012) [arXiv:1209.6083 [hep-ph]].

[26] J. Blumlein and J. Brunner, Phys. Lett. B 701, 155 (2011) [arXiv:1104.2747 [hep-ex]].

[27] B. Aubert et al. [BaBar Collaboration], Phys. Rev. Lett. 103, 081803 (2009) [arXiv:0905.4539 [hep-ex]]. 
[28] D. Babusci et al. [KLOE-2 Collaboration], Phys. Lett. B 720, 111 (2013) [arXiv:1210.3927 [hep-ex]].

[29] M. Endo, K. Hamaguchi and G. Mishima, Phys. Rev. D 86, 095029 (2012) [arXiv:1209.2558 [hep-ph]].

[30] H. Davoudiasl, H. -S. Lee and W. J. Marciano, Phys. Rev. D 86, 095009 (2012) [arXiv:1208.2973 [hep-ph]].

[31] S. Abrahamyan et al. [APEX Collaboration], Phys. Rev. Lett. 107, 191804 (2011) [arXiv:1108.2750 [hep-ex], arXiv:1108.2750 [hep-ex]].

[32] H. Merkel et al. [A1 Collaboration], Phys. Rev. Lett. 106, 251802 (2011) [arXiv:1101.4091 [nucl-ex]]. 